\title{
Soft tissue balancing in total knee arthroplasty
}

\author{
MARIA CHIARA MELONI', RUSSALKA W. HOEDEMAEKER ${ }^{1}$, BRUNO VIOLANTE ${ }^{2}$ \\ CLAUDIO MAZZOLA ${ }^{3}$
}

${ }^{1}$ Ospedale Israelitico, Rome, Italy

2 Zucchi Clinical Institutes, Monza, Italy

${ }^{3}$ Galliera Hospital, Genoa, Italy

\begin{abstract}
A good outcome in total knee arthroplasty depends on many factors: joint alignment, range of motion, patellar tracking and ligament stability. A correct soft tissue balance keeps the joint aligned in flexion and extension, and therefore constitutes the most important factor for durability of the implant. Indeed, incorrect soft tissue balancing is the primary cause of early implant failure necessitating revision surgery. Soft tissue releases, serving to correct imbalances, are performed until the flexion and extension gaps appear symmetrical and balanced. A knee is considered perfectly balanced when the flexion and extension gaps are perfectly rectangular and all the measurements are absolutely equal.
\end{abstract}

Key Words: balance, ligament, total knee arthroplasty, valgus, varus.

\section{Introduction}

A good outcome in total knee arthroplasty (TKA) depends on many factors: joint alignment, range of motion, patellar tracking and ligament stability. A correct soft tissue balance keeps the joint aligned in flexion and extension, and therefore constitutes the most important factor for durability of the implant; indeed, incorrect soft tissue balancing is the primary cause of early implant failure necessitating revision surgery.

\section{Corresponding Author:}

Maria Chiara Meloni, MD

Ospedale Israelitico, Rome, Italy

Phone: +393939574444

E-mail: chiarameloni@gmail.com
In the osteoarthritic joint, instability can be symmetrical causing cartilaginous or bony erosion without ligament changes, or asymmetrical. Asymmetrical instability, which is typical of severe osteoarthritis (OA), is characterized by the inevitable occurrence of structural soft tissue changes, serving to compensate for the deformity caused by asymmetrical bone loss. Such joint instability cannot be corrected by bone resections and implant alignment alone (Fig. 1).

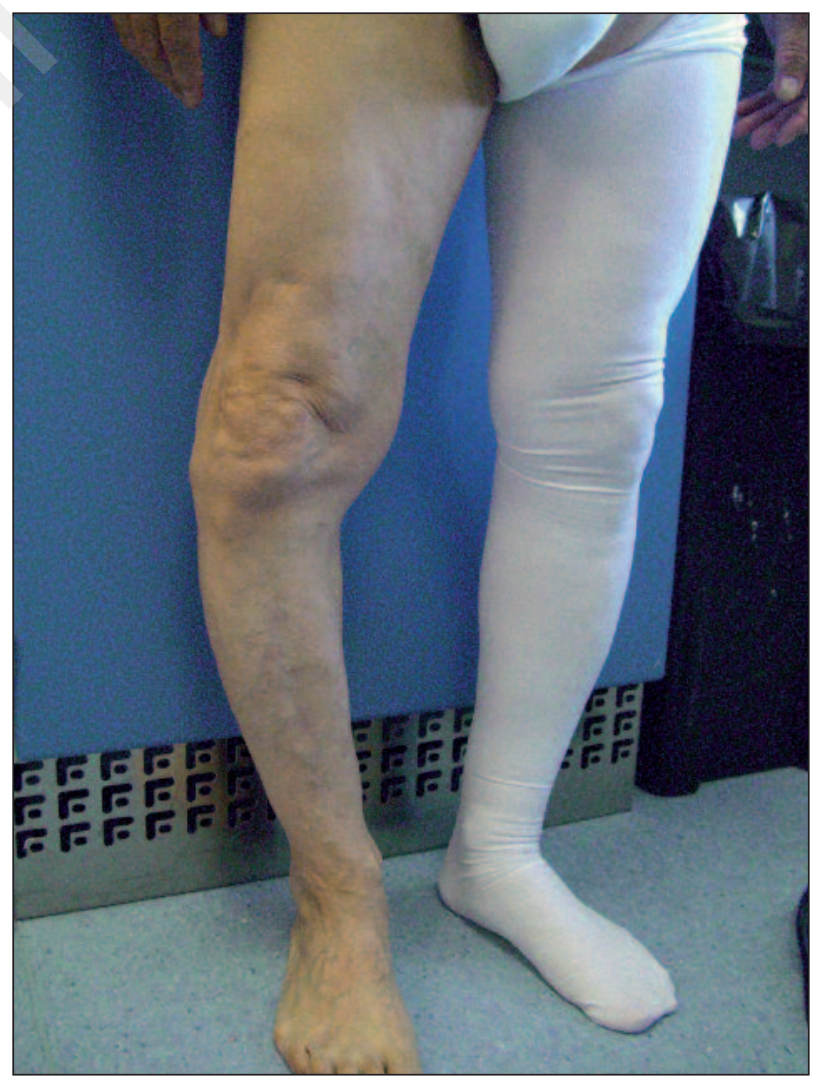

Fig, 1. Severe varus knee deformity showing both ligament and bony alterations. 
In the surgical correction of an asymmetrical joint deformity it can be necessary to perform additional procedures to ensure retention of the stretched ligamentous structures, or, if these are not sufficient, to opt for a constrained type of implant. In this regard, it is necessary to bear in mind the requirements of younger patients, in whom every effort should be made to avoid the use of constrained implants.

Each single patient should be evaluated carefully and treated individually. Accurate and thorough pre-operative planning with full-length standing radiographs of the lower limb in anteroposterior and lateral views, and axial view are indispensable for choosing the prosthesis design and tibial bone resection level $(1,2)$.

Testing for soft tissue balancing during TKA was introduced by Insall, who used spacer blocks and laminar spreaders intraoperatively to assess the extension and flexion gaps in varus and valgus stress (2).

It is recommended that all osteotomies of the tibia and femur be performed first, followed by excision of all osteophytes. Posterior femoral condylar osteophytes should be excised because they can prevent full extension and influence posterior soft tissue tension (Fig. 2). Before performing soft tissue release for balancing, it is important to remember the following points:

- The position of the prosthesis components can serve a compensatory function in an extensive soft tissue release because the flexion gap dictates rotation of the femoral component. After soft tissue balancing, the flexion space is balanced by rotating the femoral

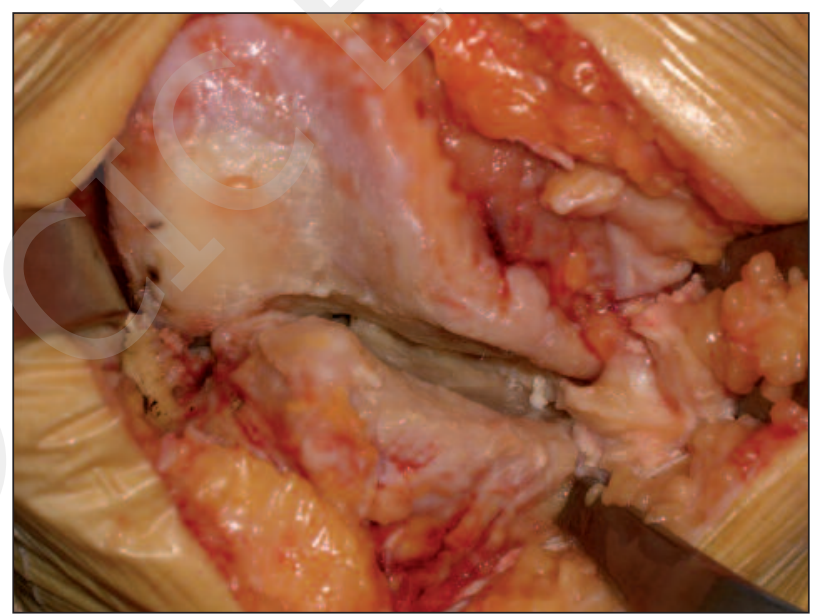

Fig. 2. Osteophytes influence soft tissue tension and do not allow proper evaluation of balancing during surgery. component to equalize the filling effect of the femoral component in flexion (Fig. 3).

- Femoral component malalignment leads to patella maltracking, flexion gap instability and anterior knee pain. In addition, a malrotated femoral component will result in an asymmetrical trapezoidal-shaped flexion gap which will increase polyethylene wear and might decrease the survival of the prosthesis.

- Tibial cut performed neutrally in the coronal plane influences the ligament balance. A bone cut performed in a few degrees of valgus is tolerated better than one performed in varus.

- In the sagittal plane, the slope can be increased to favor flexion and extension $(3,4)$.

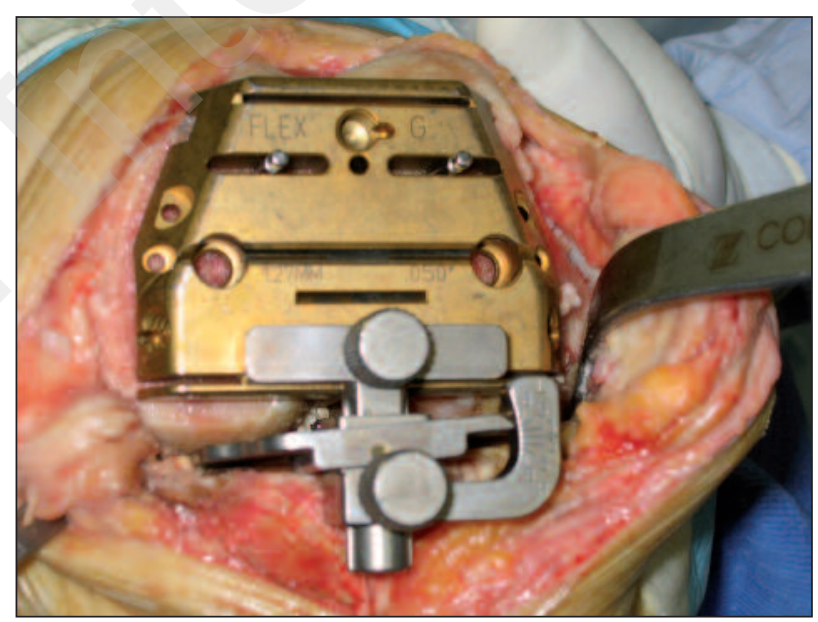

Fig. 3. Flexion space is balanced by rotating the femoral component to equalize the filling effect of the femoral component in flexion. Proper instruments are needed to reach a symmetrical gap.

\section{Varus deformity}

Varus deformity is often combined with medial soft tissue flexion contracture with lateral soft tissue laxity. Medial soft tissue serves a static stabilizing (superficial medial collateral ligament, posterior oblique ligament and posterior capsule) and a dynamic stabilizing (pes anserinus and semimembranosus tendon) function.

The critical structures on the medial side of the knee include the superficial medial collateral ligament (sMCL) fibers on the anterior aspect, and posterior structures such as the posterior oblique ligament (POL) and the semimembranosus (SM) tendon fibers that merge into the posterior capsule. 
Release of the anterior structures tends to increase the flexion gap more than the extension gap, whereas release of the more posterior elements tends to affect the extension gap more than the flexion gap. The addition of a posterior cruciate ligament (PCL) release for a posterior stabilized prosthesis increases the flexion gap. The sMCL affects both the flexion and the extension gap; however, release of only the anterior portion affects the flexion gap more than the extension gap.

The POL should be the first structure released when the knee is tight only in extension and not in flexion. Another indication for release of the POL occurs when, after release of the SMCL, the knee remains tight in extension (Fig. 4). If the knee remains tight in full extension after release of the POL, then release of the SM tendon should be considered.

Release of the SM tendon is usually only necessary in knees with significant varus coronal deformity or combined varus and flexion contracture deformity. It allows the tibia to be externally rotated and allows easier access to the posteromedial aspect of the tibia.

The pes anserinus tendons should be released only in severe varus knees; their release affects extension more than flexion.

Osteotomy of the medial epicondyle has also been reported to aid in balancing and providing exposure of the varus knee with flexion contracture. Another option for the knee with a severe varus deformity involves resection of the bone along the medial tibial plateau, with downsizing and relative lateralization of

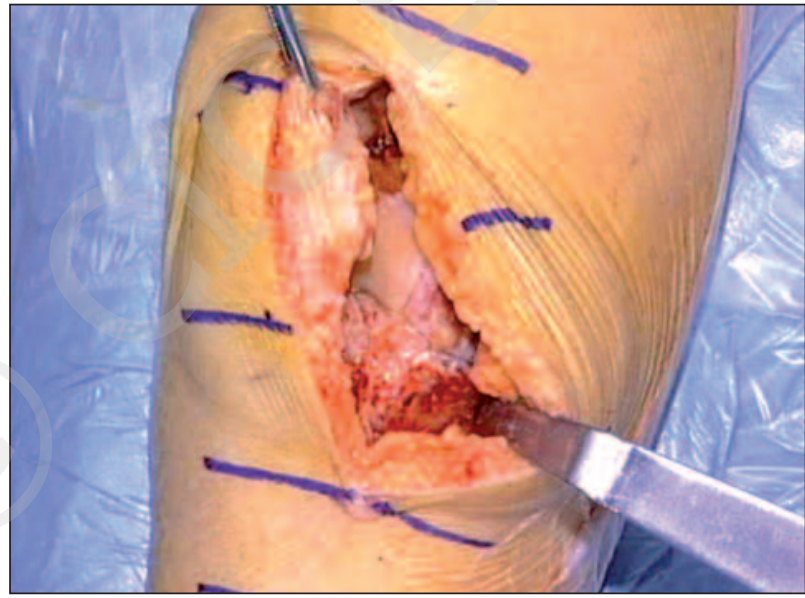

Fig. 4. Balancing of varus knee after release of both anterior and posterior structures. the tibial baseplate. The final effect is a relative medialization of the tibial tubercle, which may enhance patellar tracking.

If, after complete release of medial-sided structures, imbalance persists and the medial gap is tight, the surgeon should consider advancing the lateral collateral ligament (LCL) to correct the imbalance. This can be accomplished on the fibular side of the joint by osteotomizing the proximal fibula and advancing it distally to tighten the LCL.

In the cruciate-retaining (CR) prosthesis the tension of the PCL reduces the tibiofemoral gap in flexion, therefore a tight PCL in the CR prosthesis can produce lift-off of the polyethylene insert in flexion.

In the varus knee with a mild flexion contracture it can be sufficient to perform a soft tissue release. The posterior capsule should be released when flexion contracture persists after soft tissue balancing has equalized the medial and lateral gaps.

The capsule must be released subperiosteally from the femur. Extensive release may lead to injuries to the superior geniculate arteries, which are very difficult to reach. When a severe flexion contracture is present it is often necessary to augment the distal femoral cut, bearing in mind that this increases the risk of mid-flexion instability. Indeed, raising the joint line can provoke laxity of the collateral ligaments in mid-flexion, causing instability and patella baja $(5,6)$.

\section{Valgus deformity}

Approximately $10 \%$ of patients requiring TKA have a valgus deformity. This deformity may include bone erosion and lateral soft tissue contracture with or without medial laxity.

The lateral structures involved in the process of balancing a valgus knee are the LCL, the popliteus tendon (PT), the posterolateral corner (PLC) and the iliotibial band (ITB).

The LCL is a stabilizing structure both in flexion and in extension. It also influences the external rotation of the tibia (Fig. 5).

The PT plays a crucial stabilizing role and is an important part of the PLC of the knee. It is considered one of the main structures contributing to posterolateral 


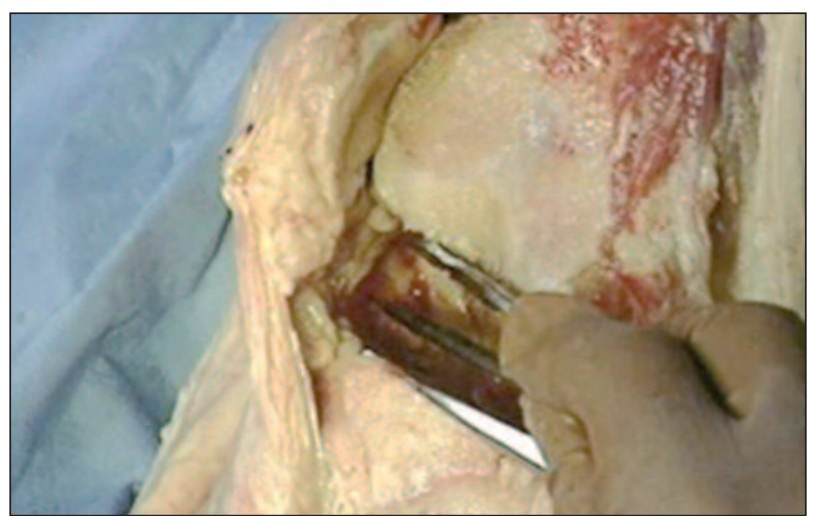

Fig. 5. Lateral collateral ligament release using the pie crusting technique in a valgus knee.

rotatory stability of the knee. It helps to unlock the knee, rotates the tibia internally on the femur, and prevents excessive external rotation of the tibia during knee flexion, among other functions. The PT should be protected because it acts as an important lateral stabilizer in higher degrees of flexion. When using a posterior substitute (PS) prosthesis, this function of the PT is critical, as it is believed to help resist dislocation of the cam-and-post mechanism when the leg is flexed and a varus force is applied.

The PLC stabilizes the knee in flexion and extension. The ITB is perpendicular to the joint line and is therefore an important lateral stabilizer only in extension. Release of the ITB must be performed only when contraction is present in extension.

In order to balance a valgus knee in extension, we first remove peripheral osteophytes and then extend the knee and distract with a laminar spreader; after this we palpate the PCL, the PLC, and the ITB with a finger or with a small Cobb elevator to identify tight structures. At this point, we can release any remnants of the PCL and release the posterolateral capsule intra-articularly using electrocautery at the level of the tibial cut surface from the PCL to the posterior border of the ITB.

If possible, the PT should be preserved, unless it is too tight, in which case we can use the so-called pie-crusting technique. This consists of transverse cutting of the arcuate ligament at the level of the tibial bone cut, and progressive lengthening of the ITB, LCL, and lateral capsule through multiple transverse stab incisions.

The gaps are then measured with a laminar spreader placed in the flexion gap. If the gaps are asymmetrical the femoral jig can be rotated, lowered or raised to create equal gaps because, when using the balanced flexion gap technique, the femoral component is rotated according to ligament tension. Therefore, bone cuts should be used to balance the knee in flexion, and controlled soft-tissue lengthening to balance the knee in extension (7-10).

According to many authors in recent years, there exists, in addition to the "measured resection technique" and the "gap balancing methodology", a third solution for alignment and soft tissue balancing, namely computer assisted surgery. CAOS (computer assisted orthopaedic surgery) can be used to balance the gaps intraoperatively, according to Insall's technique.

In conclusion, soft tissue releases, serving to correct imbalances, are performed until the flexion and extension gaps appear symmetrical and balanced. A knee is considered perfectly balanced when the flexion and extension gaps are perfectly rectangular and all the measurements are absolutely equal (7-10).

\section{References}

1. Sharkey PF, Hozack WJ, Rothman RH, et al. Why are total knee arthroplasties failing today? Clin Orthop Relat Res. 2002;404:7-13.

2. Griffin FM, Insall JN, Scuderi GR. Accuracy of soft tissue balancing in total knee arthroplasty. J Arthroplasty. 2000;15:970-973.

3. Kaipel M, Gergely I, Sinz K, et al. Femoral rotation in ligament balanced knee arthroplasty; a prospective clinical study. J Arthroplasty. 2013;28:1103-1106.

4. Mihalko WM, Whiteside LA, Krackow KA. Comparison of ligament-balancing techniques during total knee arthroplasty. J Bone Joint Surg. 2003;85A (Suppl 4):132-135.

5. Ahn JH, Back YW. Comparative study of two techniques for ligament balancing in total knee arthroplasty for severe varus knee: medial soft tissue release vs. bony resection of proximal medial tibia. Knee Surg Relat Res. 2013;25:13-18.

6. Mihalko MW, Saleh KJ, Krackow KA, et al. Soft-tissue balancing during total knee arthroplasty in the varus knee. J Am Acad Orthop Surg. 2009;17:766-774.

7. Whiteside LA. Correction of ligament and bone defects in total arthroplasty of the severely valgus knee. Clin Orthop Relat Res. 1993;288:234-245.

8. Miyasaka KC, Ranawat CS, Mullaji A. 10- to 20-year follow up of total knee arthroplasty for valgus deformities. Clin Orthop Relat Res. 1997;345:29-37.

9. Ranawat AS, Ranawat CS, Elkus M, Rasquinha VJ, Rossi R, Babhulkar S. Total knee arthroplasty for severe valgus deformity: surgical technique. J Bone Joint Surg Am. 2005;87 Suppl 1:271-284.

10. Rajgopal A, Dahiya V, Vasdev A, et al. Long-term results of total knee arthroplasty for valgus knees: soft-tissue release technique and implant selection. J Orthop Surg (Hong Kong). 2011; 19:60-63. 
negative Lachman and pivot shift tests. All other ligamentous structures and any other surgical steps are of little, if any, value.

Since then many things have changed: several arthroscopic ACL reconstruction techniques have been developed, allowing better and better reproduction of the native ACL: anatomical, non-anatomical, singlebundle, double-bundle, in-out, out-in, all-inside. The use of hamstrings has become increasingly widespread and rehabilitation protocols have evolved, and so on. All these changes have radically influenced the approach to the ACL deficient knee, and resulted in a significant improvement of clinical results. However, most authors report the presence of a certain degree of pivot shift at follow-up (a sign of persistent instability) and this issue has been identified as the one that researchers should focus on in the future (2). Among the possible reasons for the inadequacy of current IR techniques to perfectly restore knee stability following an ACL reconstruction, some authors have cited failure to recognize and/or failure to adequately treat injuries of the anterolateral corner, which can significantly affect the severity of the pivot shift and were specifically addressed, in the past, by ERs (3). It may therefore be opportune and beneficial to reconsider ERs in the light of the substantial changes in the approach to ACL surgery that have taken place over the past two decades, and of recent acquisitions in knee biomechanics, possibly revisiting the statements validated in Snowmass.

In this paper, we will present and examine the main issues discussed and the statements consensually validated in Snowmass to evaluate whether they are still valid or, instead, need to be totally or partially revised. Moreover, just as the experts on that occasion concluded by asking questions and offering suggestions for future research, we will attempt to highlight new advances and provide indications, drawing on the most recent studies, research and publications.

\section{The Big Five}

In Snowmass, five study groups were formed to discuss, in depth, the following topics:
- The biomechanics of the extra-articular reconstruction;

- Extra-articular reconstruction in the skeletally immature knee;

- Extra-articular reconstruction as the primary procedure in the ACL deficient knee;

- Extra-articular reconstruction as a secondary support procedure in conjunction with intra-articular reconstruction in the acute anterior cruciate defi $\bullet$ cient knee;

- Extra-articular reconstruction as a secondary support procedure in conjunction with intra-articular reconstruction in the chronic anterior cruciate deficient knee.

Each of these groups presented its deliberations to the entire workshop which, after discussion, concluded with a formal consensus validation process.

Below, the content of the statements issued by the groups and the general assembly is summarized and discussed. Since the experts were not able to undertake a consensual validation process for topic 4 , due to the limited literature available, topics 4 and 5 are discussed together.

\section{The biomechanics of the extra-articular reconstruction}

Statements 1 and 2: ERs cannot fully restore normal biomechanics in an ACL deficient knee.

This point was consensually validated and has been confirmed by more recent studies. ERs reduce tibial rotation with a limited effect on anterior tibial translation (4).

Statement 3: ERs are biomechanically less efficacious than IRs.

This statement is in line with statements 1 and 2, and it remains valid.

Statement 4: Intra-articular repairs and reconstructions can be partially stress-protected by ERs.

A study by Draganich et al. (5) confirmed the findings of Engebretsen et al. (6), which showed that ERs can share the load applied to a reconstructed ACL during rotational and translational stresses. 
Statement 5: ERs can reduce pivot shift, initially at least, and can reduce anterior tibial translation, but only to the extent that they can control lateral tibial plateau.

This statement has been validated by some recent studies (4).

Statement 6: At present there is insufficient biomechanical data to recommend an optimal extra-articular attachment for an ER.

To the best of our knowledge, no further studies have been published on this subject. Therefore this statement should be considered valid.

Statements 7, 8 and 9: Grafts used for ERs are weaker than normal ACL.

Decreased strength of the graft and initial fixation, as well as increased stress to the graft due to the absence of the ACL, could explain high failure rate of ERs when used alone in ACL deficient knees.

\section{Extra-articular reconstruction in the skeletally immature knee}

Statement 1: Bony avulsions, which mostly occur at the tibial attachment, should be surgically repaired. The use of ERs is generally discouraged unless there is associated secondary restraint injury.

This statement, which was unanimously validated, remains valid. However, as when severe pivot shifts are detected, injury to secondary restraints should be considered, especially in the anterolateral compartment. In such cases, careful examination and repair of the lateral capsule could be recommended as an adjunctive procedure (7).

Statement 2: Partial tear and tears resulting in mild instability should be treated conservatively.

This statement, which was unanimously validated, is still valid. The problem is how to diagnose a partial tear. In our opinion, a partial tear not affecting knee stability can be suspected in the presence of negative laxity tests and ACL abnormalities at arthroscopy or on MRI. On the contrary, in the presence of positive laxity tests any tear of the ACL should be considered total and treated accordingly (8).
Statement 3: ACL injuries in Tanner stages III and IV should be treated as in adults.

This statement has been confirmed by several studies published subsequently (9).

Statement 6: ERs as ACL substitutes should be avoided; however, if performed, only techniques that avoid the risk of peripheral damage to the growth plate by fixation devices and drill holes should be used.

It is interesting to note that the experts did not close the door to possible use of these techniques in selected cases.

Statement 8: There is little or no role for ERs to replace or support the ACL. However, the surgeon may consider anatomical repair of secondary restraints in (more severe) level 3 and 4 injuries.

The experts acknowledged the role of secondary restraints in determining severe instability and again considered the possibility of anatomical repair of secondary restraints in such cases.

\section{Extra-articular reconstruction as the primary procedure in the ACL deficient knee}

Statement 1: Primary ERs have an only limited role as it is well accepted that isolated ERs are biomechanically inferior to IRs and therefore should not be performed.

This statement is still accepted as valid and we fully agree with it (10).

Statement 4: Suitable cases for ERs might include older, low demanding patients, patients with degenerative joint diseases and synovitis and patients exhibiting combinations of misalignment in whom a reconstruction with a high tibial osteotomy might be indicated.

The tendency (based on subsequent studies of patients older than 40-50 years) to use less invasive and less arthrogenic surgical and rehabilitation techniques has further reduced the indications for isolated ERs in the ACL deficient knee (11). Nowadays, there are few cases in which an isolated ER might be performed rather than an arthroscopically assisted IR with hamstrings, even in older, low demanding or arthritic patients. 
Extra-articular reconstruction as a secondary support procedure in conjunction with intra-articular reconstruction in the acute and chronic ACL deficient knee

These scenarios are the most important as they are the ones still most debated among surgeons. They are therefore discussed in more detail here.

The group which discussed the use of ERs in conjunction with IRs in the acute ACL deficient knee was unable to undertake a consensual validation process due to the limited available literature at that time. The authors of the aforementioned booklet (1) therefore conducted a further review of the literature and concluded that combined reconstruction in acute ACL tears was an area that still needed extensive research, especially to address the role of secondary restraints, the possible risk of over-constraining the knee, and the influence of rehabilitation on combined procedures. However, even though the results of this review of the literature were in actual fact inconclusive, some authors have stated that the combined procedure should be utilized in the grossly unstable knee with severe anterolateral rotational instability and that the post-operative regimen must include immediate weight-bearing and range-of-motion (ROM) exercises. A similar recommendation was made by Wilson and Scranton (12), who used the semitendinosus as an intra-articular graft, and by Noyes and Barber (13), who used an allograft as ACL substitute and an accelerated rehabilitation regimen. Conversely, authors who did not recommend the use of ERs mostly used the bone-patellar tendon-bone (BPTB) graft followed by cast immobilization.

In conclusion, over the 80 s and the 90 s most authors came to recognize the value of ERs used in association with IRs, in acute cases and providing the IR was performed with a graft other than BPTB and the procedure was followed by an immobilization-free rehabilitation protocol.

The work of the study group evaluating ERs as a secondary support procedure in conjunction with IRs in the chronic ACL-deficient knee was designed as a professional inquiry and not a pure scientific investigation. In short, the experts were asked to give their personal opinions rather than provide information extracted from in-depth analysis of the current literature. And, in fact, the conclusions reflect this preliminary bias. On the basis of their analysis, the experts made the following statements:

- Clinically, in the chronic ACL deficient knee, increased secondary restraint laxity appears to occur, probably as a result of re-injury and possibly due to progressive stretching related to overuse. Increased laxity of the secondary restraints may have an adverse effect on increase in rotation of the tibia.

- Extra-articular procedures may benefit the abnormal tibial translation present in the ACL-deficient knee and may decrease the incidence of meniscal tears.

Moreover, the experts suggested that the term anterolateral instability, which was used in the literature as a synonym for ACL insufficiency, should be used to identify a different condition, possibly related to a more complex injury involving both the ACL and other capsule-ligamentous structures, given that absence of the ACL in isolation results in abnormal tibial translation with an only nominal effect on rotation.

The above statements and suggestion seem to show that some of the experts believed anterolateral instability and pivot shift, which severely affect knee function, to be related to a more complex injury, involving several structures, rather than to an isolated ACL tear, and that ERs in conjunction with IRs could more comprehensively address pathological laxity; accordingly they acknowledged that these procedures could possibly play a decisive role.

The experts then went on to analyze various concerns, issues and problems. This chapter contains no references, which seems to indicate that the experts were expressing their own opinions rather than consolidated, well-documented and scientifically validated points.

The main problems arising from the discussion were related to the identification of a desirable isometric point of fixation on the lateral femoral condyle, the risk of increasing compression forces on the lateral compartment and of over-constraining the knee in internal rotation, the risk of abnormal varus and posterolateral laxity, the risk of loss of full extension and the possibility of patellofemoral problems. At the end of the discussion the experts stated that the benefits of a lateral reconstruction might not justify the increased 
morbidity related to the additional procedure, and that adding ER would necessitate a rigid post-operative immobilization, leading to further problems with recovery of motion and muscle function, in contrast to an isolated BPTB graft which permitted early motion. In conclusion, the experts stated that lateral ER procedures have limited application, i.e. they can be used only as a means of augmenting high-strength IRs. In these very limited circumstances, the lateral ERs that appear reasonable are those in which a static restraint is created between the area of Gerdy's tubercle and the appropriate location on the lateral femur, given that there is no absolute isometric site.

Finally, as indications for future research, the experts suggested studies on articular pressures (including patellofemoral joint pressures), studies on abnormal translation and rotation of the knee as a result of various ligamentous tears and of various surgical reconstruction procedures, as well as long-term follow-up studies of patients undergoing ACL reconstruction with or without ER.

\section{Discussion}

The statements, remarks and conclusions of the groups of experts warrant some comments.

We have no argument with the experts' analysis of the biomechanics of the ACL and the concept of rotatory instability. Studies performed after the Snowmass meeting confirmed that the pivot shift, the test designed to evaluate and grade anterolateral instability, is only mildly positive as a result of an isolated tear of the ACL, whereas it is significantly increased by a tear of the anterolateral structures, namely the anterolateral ligament (3). Recent cadaver studies, using sophisticated methods of investigation, have confirmed the role of secondary restraints in producing the pivot shift phenomenon in the presence of an ACL tear (7). Therefore, IRs alone, regardless of the type of graft used, may be unable to fully compensate for the pathological laxity and ERs could be biomechanically justified.

The aspect of the experts' work that we strongly criticize is their analysis of concerns, issues and problems on the basis of personal impressions and experience rather than objective data supported by the literature; in fact, they cited no studies considering the clinical relevance of the possible increase in pressure in the lateral compartment, the weakness of the lateral structures, the development of a varus deformity, the loss of ROM or the occurrence of late OA. Moreover, as the use of hamstrings in IRs spread and became more popular among knee surgeons, and accelerated rehabilitation became the standard approach, even in combined procedures, all the concerns and issues about the increased risk of delayed recovery of muscle function, the loss of ROM, and the development of arthrofibrosis were progressively overcome, as confirmed by the results of the studies performed in the centers that continued to use ERs in association with IRs, using a more compliant hamstring graft and an accelerated rehabilitation protocol (14-18).

It seems to be that the experts at that time were extremely concerned about possible complications related to any surgical procedure performed in association with an intra-articular ACL reconstruction. This is understandable if we consider the techniques that were most popular at the time, which included the use of BPTB, even in acute cases, performed in an open fashion, often after a diagnostic arthroscopy, and followed by a very cautious rehabilitation protocol with several weeks of immobilization in a cast or brace. In these circumstances, any additional surgical procedure, even one mildly increasing the morbidity of the reconstruction, could have devastating effects on a joint already submitted to a very invasive procedure. However, none of the elements used by the authors to support their hypothesis of possible increased risks, contraindications, complications is supported by scientific evidence. To the best of our knowledge, no studies have reported negative clinical effects of increased pressure on the lateral compartment, increased morbidity, an increased risk of arthrofibrosis, a delayed functional recovery, or increased laxity of the lateral compartment leading to a genu varum, when an ER was associated with a correctly performed IR that complied with the modern principles of surgical and rehabilitation techniques. Moreover, authors (especially in Europe) who continued to combine ERs and IRs using hamstrings reported better clinical results, improved rotatory stability and higher Tegner scores, 
with no increased rate of complications, radiological signs of OA or arthrofibrosis. Moreover, all these authors continue to recommend the use of ERs in association with ACL reconstruction with hamstrings in patients with severe instability (pivot shift ++ or +++ ), female athletes, high-level/high-risk athletes and revision surgery cases (18-20).

\section{Conclusions}

The Snowmass meeting was a milestone event as regards efforts to clarify the role of ERs in ACL-deficient knees, making an outstanding contribution to the definition of these techniques, their biomechanical effects and their role in rotatory instability. The experts who gathered in Snowmass recognized that the clinical impact of an ACL tear can be significantly increased by tears of secondary restraints, such as the ligamentous and capsular structures of the lateral compartment, which therefore need to be properly recognized and treated. However, their conclusions, reached at the end of a comprehensive discussion, appear to be totally at odds with their premises, given that they failed to identify any advantage of secondary restraint reconstruction/repair, performed in association with the ACL reconstruction, over intra-articular reconstruction alone. However, their conclusions seemed to be based on subjective feelings or personal experiences rather than on an objective review of the literature and, overall, on knowledge and surgical and rehabilitation techniques of that time. These experts, who apparently did not envisage an evolution of surgical techniques and rehabilitation approaches, instead of recommending further studies to develop less invasive and more effective techniques for using both IRs and ERs in association, encouraged abandonment of ERs (isolated or associated with IRs), both in adult and in adolescent patients.

Today, we can say that the conclusions of the Snowmass meeting were misleading or at least misinterpreted and, overall, that they are not supported by any of the more recent studies on this subject. Moreover, they were based on surgical techniques and rehabilitation procedures that are no longer in use in modern ACL surgery. For these reasons, we suggest that it would today be opportune to reconsider, without prejudice, the role of ERs, updating all aspects of these procedures in the hope that they might open the way for a more comprehensive understanding of the pathoanatomy of knee instability following an ACL tear, and a more rational surgical approach to the condition.

\section{References}

1. Pearle A, Bergfeld J. Extraarticular reconstruction in ACL deficient knee. Human Kinetics, Chicago (IL). 1992.

2. Ayeni OR, Chabal M, Tran MN, Sprague S. Pivot shift as an outcome measure for ACL reconstruction: a systematic review. Knee Surg Sports Traumatol Arthrosc. 2012;20:767777.

3. Tanaka M, Vyas D, Moloney G, Bedi A, Pearle AD, Musahl V. What does it take to have a high-grade pivot shift? Knee Surg Sports Traumatol Arthrosc. 2012;20:737-742.

4. Monaco E, Labianca L, Conteduca F, De Carli A, Ferretti A. Double bundle or single bundle plus extraarticular reconstruction in ACL reconstruction? A CAOS study. Knee Surg Sports Traumatol Arthrosc. 2007;15:1168-1174.

5. Draganich LF, Reider B, Ling M, Samuelson M. An in vitro study of an intraarticular and extraarticular reconstruction in the anterior cruciate ligament deficient knee. Am J Sports Med. 1990;18:262-266.

6. Engebretsen L, Lew WD, Lewis JL, et al. The effect of iliotibial tenodesis on intraarticular graft forces and knee joint motion. Am J Sports Med. 1990;18:169-176.

7. Monaco E, Ferretti A, Labianca L, et al. Navigated knee kinematics after cutting of ACL and its secondary restraints. Knee Surg Sports Traumatol Arthrosc. 2012;20:870-877.

8. Furman W, Marshall JL, Girgis FG. The anterior cruciate ligament. A functional analysis based on post mortem studies. J Bone Joint Surg. 1976;58A:179-185.

9. Kaeding CC, Flanigan D, Donaldson C. Surgical techniques and outcomes after anterior cruciate ligament reconstruction in preadolescent patients. Arthroscopy. 2010;26:1530-1538.

10. Garcia R Jr, Brunet ME, Timon S, Barrack RL. Lateral extraarticular reconstruction: long-term patient outcome and satisfaction. J South Orthop Assoc. 2000; 9:19-23.

11. Desai N, Björnsson H, Samuelsson K, Kalsson J, Forssblad M. Outcomes after ACL reconstruction with focus on older patients: results from The Swedish National Anterior Cruciate Ligament Register. Knee Surg Sports Traumatol Arthrosc. 2014;22:379-396.

12. Wilson WJ, Scranton PE Jr. Combined reconstruction of the anterior cruciate ligament in competitive athletes. J Bone Joint Surg Am. 1990;72:742-748.

13. Noyes FR, Barber SD. The effect of an extra-articular procedure on allograft reconstructions for chronic ruptures of the anterior cruciate ligament. J Bone Joint Surg Am. 1991;73: 882-892.

14. Goertzen M, Schulitz KP. Comparison of combined extra and intra-articular stabilization versus isolated arthroscopic semitendinosus repair after rupture of the anterior cruciate ligament. Sportverlez Sportschaden. 1993;7:7-12.

15. Goertzen M, Schulitz KP. Isolated intraarticularplasty of the semitendinosus or combined intra- and extra-articular plasty 
in chronic anterior laxity of the knee. Rev Chir Orthop Reparatrice Appar Mot. 1994,80:113-117.

16. Trichine F, Alsaati M, Chouteau J, Moyen B, Bouzitouna M, Maza R. Patella tendon autograft reconstruction of the anterior cruciate ligament with and without lateral plasty in advanced stage chronic laxity. A clinical, prospective, randomized single-blind study using passive dynamic X-rays. Knee. 2014;21:58-65.

17. Pernin J, Verdonk P, Si Selmi TA, Massin P, Neyret P. Longterm follow up of 24.5 years after intra-articular anterior cruciate reconstruction with lateral extra-articular augmentation. Am J Sports Med. 2010; 38:1094-1102.

18. Vadalà AP, Iorio R, De Carli A, Bonifazi A, Iorio C, Gatti A, Rossi C, Ferretti A. An extra-articular procedure improves the clinical outcome in anterior cruciate ligament reconstruction with hamstrings in female athletes. Int Orthop. 2013;37:187-192.

19. Trojani C, Beaufils P, Burdin G, Bussière C, Chassaing V, Djian P, Dubrana F, Ehkirch FP, Franceschi JP, Hulet C, Jouve F, Potel JF, Sbihi A, Neyret P, Colombet P. Revision ACL reconstruction: influence of lateral tenodesis. Knee Surg Sports Traumatol Arthrosc. 2012;20:1565-1570.

20. Ferretti A, Conteduca F, Monaco E, De Carli A, D’Arrigo C. Revision anterior cruciate ligament reconstruction with doubled semitendinosus and gracilis tendons and lateral extraarticular reconstruction. J Bone Joint Surg Am. 2006;88: 2373-2379. 